\title{
The cosmic far-infrared background buildup since redshift 2 at 70 and 160 microns in the COSMOS and GOODS fields
}

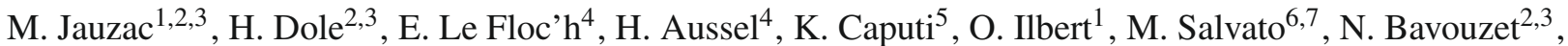 \\ A. Beelen ${ }^{2,3}$, M. Bétherminn ${ }^{2,3}$, J.-P. Kneib ${ }^{1}$, G. Lagache ${ }^{2,3}$, and J.-L. Puget ${ }^{2,3}$ \\ ${ }^{1}$ Laboratoire d'Astrophysique de Marseille, Université de Provence, CNRS, 13388 Marseille Cedex 13, France \\ e-mail: mathilde.jauzac@oamp.fr \\ 2 Univ Paris Sud, Institut d'Astrophysique Spatiale (UMR 8617), Bât. 121, 91405 Orsay, France \\ 3 CNRS, Orsay 91405, France \\ 4 IRFU, SAp, CNRS, Saclay, Bât. 709, Orme des merisiers, 91191 Gif-sur-Yvette, France \\ 5 SUPA, Institute for Astronomy, The University of Edinburgh, Royal Observatory, Edinburgh - EH9 3HJ, UK \\ ${ }^{6}$ California Institute of Technology, MC 105-24, 1200 East California Boulevard, Pasadena, CA 91125, USA \\ 7 Max-Planck Institute for Plasma Physics \& Cluster of Excellence, Boltzmann Strasse 2, 85748 Garching Germany \\ Received 19 July 2010 / Accepted 31 August 2010
}

ABSTRACT

\begin{abstract}
Context. The cosmic far-infrared background (CIB) at wavelengths around $160 \mu \mathrm{m}$ corresponds to the peak intensity of the whole extragalactic background light, which is being measured with increasing accuracy. However, the build up of the CIB emission as a function of redshift is still not well known.

Aims. Our goal is to measure the CIB history at $70 \mu \mathrm{m}$ and $160 \mu \mathrm{m}$ at different redshifts, and provide constraints for infrared galaxy evolution models.

Methods. We used deep Spitzer $24 \mu \mathrm{m}$ catalogs complete to about $80 \mu \mathrm{Jy}$ with spectroscopic and photometric redshift identifications, derived using the GOODS and COSMOS deep infrared surveys covering 2 square degrees total. After cleaning the Spitzer/MIPS $70 \mu \mathrm{m}$ and $160 \mu \mathrm{m}$ maps of detected sources, we stacked the far-IR images at the positions of the $24 \mu \mathrm{m}$ sources in different redshift bins. We measured the contribution of each stacked source to the total 70 and $160 \mu \mathrm{m}$ light, and compared with model predictions and far-IR measurements obtained for Herschel/PACS data of smaller fields.

Results. We detect components of the 70 and $160 \mu \mathrm{m}$ backgrounds in different redshift bins up to $z \sim 2$. The contribution to the CIB reaches a maximum at $0.3 \leq z \leq 0.9$ at $160 \mu \mathrm{m}$ (and $z \leq 0.5$ at $70 \mu \mathrm{m}$ ). A total of $81 \%(74 \%)$ of the $70(160) \mu \mathrm{m}$ background was emitted at $z<1$. We estimate that the AGN contribution to the far-IR CIB is less than about $10 \%$ at $z<1.5$. We provide a comprehensive view of the CIB buildup at 24, 70, 100 and $160 \mu \mathrm{m}$.

Conclusions. We find that IR galaxy models predicting a major contribution to the CIB from sources at $z<1$ agree with our measurements, while our results exclude other models that predict a peak of the background at higher redshifts. The consistency of our results with those obtained by the direct study of Herschel far-IR data at $160 \mu \mathrm{m}$ confirms that the stacking analysis method is a valid approach to estimate the components of the far-IR background using prior information about resolved mid-IR sources.
\end{abstract}

Key words. cosmology: observations - diffuse radiation - galaxies: evolution - galaxies: starburst - galaxies: active infrared: diffuse background

\section{Introduction}

The extragalactic background light (EBL) is the relic emission of galaxy formation and evolution, i.e., produced by star formation and accretion processes (according to this definition, the cosmic microwave background due to recombination at redshift $z \sim 1100$ is not part of the EBL). The EBL spectrum peaks in the far-infrared (FIR), where it is commonly refered to as the cosmic infrared background (CIB) (Puget et al. 1996; Hauser et al. 1998; Hauser \& Dwek 2001; Kashlinsky 2005; Dole et al. 2006). The EBL and the CIB encode the emission processes of structure formation, and can thus be used to constrain the photon budget of the cooling processes leading the baryons to fall within the dark matter halos and form galaxies. The measurements of the EBL level and structure bring thus one of the many useful constraints for the galaxy formation and evolution models.

The CIB spectral energy distribution is measured with increasing accuracy (for instance in the FIR and submillimetre regime: Puget et al. 1996; Aharonian et al. 2006; Dole et al. 2006; Bethermin et al. 2010a), but little is known about its history, i.e., its buildup as a function of redshift. This missing information should help us to constrain galaxy evolution models, and more clearly understand the physics of blazars, whose high-energy photons interact with the CIB along the line of sight (e.g., Aharonian et al. 2007; Albert \& Magic Collaboration 2008; Raue et al. 2009; Kneiske \& Dole 2009).

The history of the CIB buildup can be derived by integrating the luminosity functions of galaxies as a function of redshift (neglecting other sources of diffuse emission and thus assuming that the CIB is due to galaxies). This is a very difficult task in practice, since high-redshift luminosity functions have not yet been measured at wavelengths close to the peak of the CIB (near $160 \mu \mathrm{m})$ but instead in the mid-infrared range (e.g. Le Floc'h et al. 2005; Caputi et al. 2007), or only in the local universe (Soifer \& Neugebauer 1991; Takeuchi et al. 2006). This situation is about to change with the latest Spitzer surveys and the ongoing deeper Herschel surveys (Magnelli et al. 2009; Clements et al. 2010; Dye et al. 2010).

Two breakthroughs have been made by using COSMOS and GOODS surveys. Firstly, using about 30000 Spitzer $24 \mu \mathrm{m}$ 
selected sources with accurate photometric redshifts (Ilbert et al. 2009), Le Floc'h et al. (2009) were able to measure the $24 \mu \mathrm{m}$ background buildup with redshift (e.g. their Figs. 7 to 9). They furthermore show that the redshift information is crucial when comparing data with the models, since it helps breaking degeneracies in the model parameters. Secondly, using the redshift identification of Herschel/PACS 100 and $160 \mu \mathrm{m}$ sources, Berta et al. (2010) were able to measure the CIB build up in four redshift bins, in the $140 \operatorname{arcmin}^{2}$ GOODS-N field (an area about 40 times smaller than used in this analysis).

In this paper, we measure the $70 \mu \mathrm{m}$ and $160 \mu \mathrm{m}$ CIB history since $z=2$, by performing a stacking analysis of galaxies detected at $24 \mu \mathrm{m}$ (a good proxy for the $160 \mu \mathrm{m}$ CIB population, e.g. Dole et al. 2006; Bethermin et al. 2010a) in the Spitzer data of the GOODS and COSMOS fields ${ }^{1}$. This approach complements over a large area the studies with Herschel in Berta et al. (2010) at 100 and $160 \mu \mathrm{m}$.

\section{Data and sample}

\subsection{GOODS data}

The data were acquired by the MIPS imaging photometer at $24 \mu \mathrm{m}, 70 \mu \mathrm{m}$, and $160 \mu \mathrm{m}$ (Rieke et al. 2004) onboard the Spitzer infrared space telescope (Werner et al. 2004), and come from the GOODS team (Chary et al. 2004) and guaranteed time observations (Papovich et al. 2004; Dole et al. 2004) of the Chandra Deep Field South (CDFS) and the Hubble Deep Field North (HDFN). Papovich et al. (2004) extracted a catalog at $24 \mu \mathrm{m}$, with $80 \%$ completeness at $80 \mu \mathrm{Jy}$. We used a sample of 1349 galaxies with $24 \mu \mathrm{m}$ flux densities $S_{24} \geq 80 \mu \mathrm{Jy}$, located in the two GOODS fields, north and south, for a total area of 291 sq. arcmin (Caputi et al. 2006; Caputi et al. 2007). The galaxies were completely identified, and redshifts were determined for all of them, with more than $45 \%$ of spectroscopic redshifts. Active galactic nuclei (AGNs) were distinguished from star-forming systems using X-ray data and near-infrared (3.6 to $8 \mu \mathrm{m}$ ) colors: we identified 136 AGNs among 1213 starforming systems (Caputi et al. 2007). To determine the contribution of mid-infrared galaxies to the far-infrared background in redshift slices, we divided the $24 \mu \mathrm{m}$ sample into four redshift bins: $0<z<0.65$ with 317 sources (of which 9 AGNs); $0.65<z<1.3$ with 575 sources (45 AGNs); $1.3<z<2$ with 259 sources ( 38 AGNs); and $z>2$ with 198 sources (44 AGNs). These bins were chosen to maximize the number of sources present in each bin, while keeping the $\Delta z$ width constant.

\subsection{COSMOS data}

The Cosmic Evolution survey (COSMOS) data were acquired by MIPS at 24, 70, and $160 \mu \mathrm{m}$. The $24 \mu \mathrm{m}$ observations of the COSMOS field is part of two general observer programs (PI Sanders): G02 (PID 20070) carried out in January 2006, and G03 (PID 30143) carried out in 2007. We used a total net area of 1.93 square degrees. Le Floc'h et al. (2009) extracted a catalogue at $24 \mu \mathrm{m}$ and provided us with a sample of 32840 galaxies found to have $24 \mu \mathrm{m}$ flux densities $S_{24} \geq 80 \mu \mathrm{Jy}$. The completeness limit is about $90 \%$ at this level. (Note that the survey sensitivities of the COSMOS and GOODS fields are similar at 24, 70 , and $160 \mu \mathrm{m})$. The $24 \mu \mathrm{m}$ galaxies were completely identified, and redshifts were derived by Ilbert et al. (2009) and

\footnotetext{
${ }_{1}$ Our results are available online at http://www.ias.u-psud.fr/ irgalaxies/
}

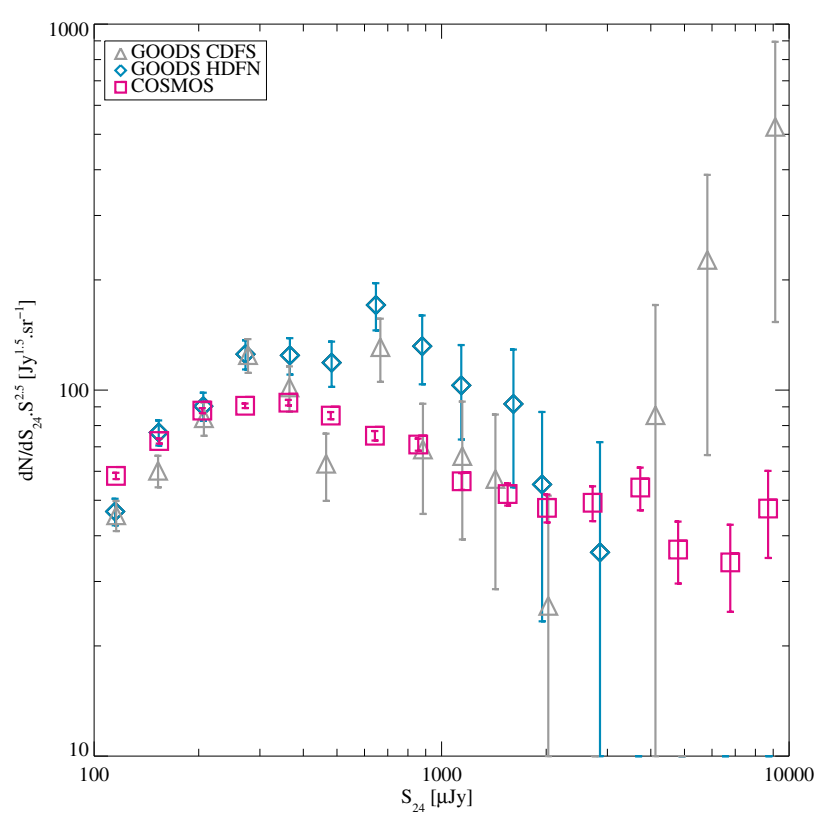

Fig. 1. Number counts at $24 \mu \mathrm{m}$ in the GOODS HDFN (blue diamond), GOODS CDFS (gray triangle), and COSMOS (pink square) fields. The errors bars used only iaccount for Poisson statistics and not cosmic variance.

Salvato et al. (2009) for the optically and X-ray selected sources of the COSMOS field respectively. We used the Salvato et al. (2009) photometric redshift catalogue created using the Cappelluti et al. (2009) X-ray source catalogue, optically matched by Brusa et al. (2007) and Brusa et al. (2010), to identify the AGNs in the COSMOS field (Le Floc'h et al. 2009). We note that the X-ray flux limits used in the soft $(0.5-2 \mathrm{keV})$, hard $(2-10 \mathrm{keV})$, or ultra-hard $(5-10 \mathrm{keV})$ bands are $5 \times 10^{-16}, 3 \times 10^{-15}$, and $5 \times 10^{-15} \mathrm{erg} \mathrm{cm}^{-2} \mathrm{~s}^{-1}$, respectively (Cappelluti et al. 2007, 2009; Salvato et al. 2009). We complement this sample with sources found to have a power-law SED (Alonso-Herrero et al. 2006) in the redshift range $1.5<z<2.5$ using IRAC colors (at lower and higher redshifts, the colors can be contaminated by the PAH or stellar bumps) in the same way as for the GOODS sample. We obtained 1668 sources (1115 X-ray sources, 553 power-law sources) detected at $24 \mu \mathrm{m}$ and identified as AGNs for 31172 star forming systems.

Since the COSMOS sample is larger than that of GOODS, we used 14 redshift bins, described in Table 1 . The source statistics in these fields is summarized in Fig. 1, showing the number counts of the GOODS survey (CDFS and HDFN) as well as the COSMOS field, corrected for incompleteness. The errors bars used only include Poisson statistics, and not cosmic variance, and are thus likely underestimated. There is no evidence of a relative major over- or under-density, except maybe a slight overdensity in the HDFN around $1 \mathrm{mJy}$, which has a negligible contribution to the total background.

\section{Analysis}

\subsection{Stacking analysis}

To estimate the contribution of mid-infrared galaxies to the $70 \mu \mathrm{m}$ and $160 \mu \mathrm{m}$ background, we used a stacking analysis ${ }^{2}$

2 The IAS stacking library, written in IDL, is publicly available at http://www.ias.u-psud.fr/irgalaxies, cf. Bavouzet (2008) and Bethermin et al. (2010a). 
Table 1. Number of $24 \mu \mathrm{m}$ sources per redshift bins for the COSMOS field.

\begin{tabular}{lccccccc}
\hline \hline$z$ bin & $0<z<0.15$ & $0.15<z<0.3$ & $0.3<z<0.45$ & $0.45<z<0.6$ & $0.6<z<0.75$ & $0.75<z<0.9$ & $0.9<z<1.05$ \\
\hline$N_{\text {tot }}$ & 2083 & 1559 & 2853 & 2201 & 3225 & 3590 & 3478 \\
$N_{\text {AGNs }}$ & 34 & 32 & 74 & 48 & 88 & 110 & 123 \\
\hline$z$ bin & $1.05<z<1.2$ & $1.2<z<1.35$ & $1.35<z<1.5$ & $1.5<z<1.65$ & $1.65<z<1.85$ & $1.85<z<2.05$ & $z>2.05$ \\
\hline$N_{\text {tot }}$ & 2670 & 1401 & 2044 & 1311 & 1519 & 2073 & 2833 \\
$N_{\text {AGNs }}$ & 83 & 76 & 55 & 225 & 232 & 200 & 288 \\
\hline
\end{tabular}

Notes. $N_{\text {tot }}$ is the total number of galaxies used in the stacks, and $N_{\mathrm{AGNS}}$ is the number of sources identified as AGNs that were used in the stacks to estimate the AGN contribution.

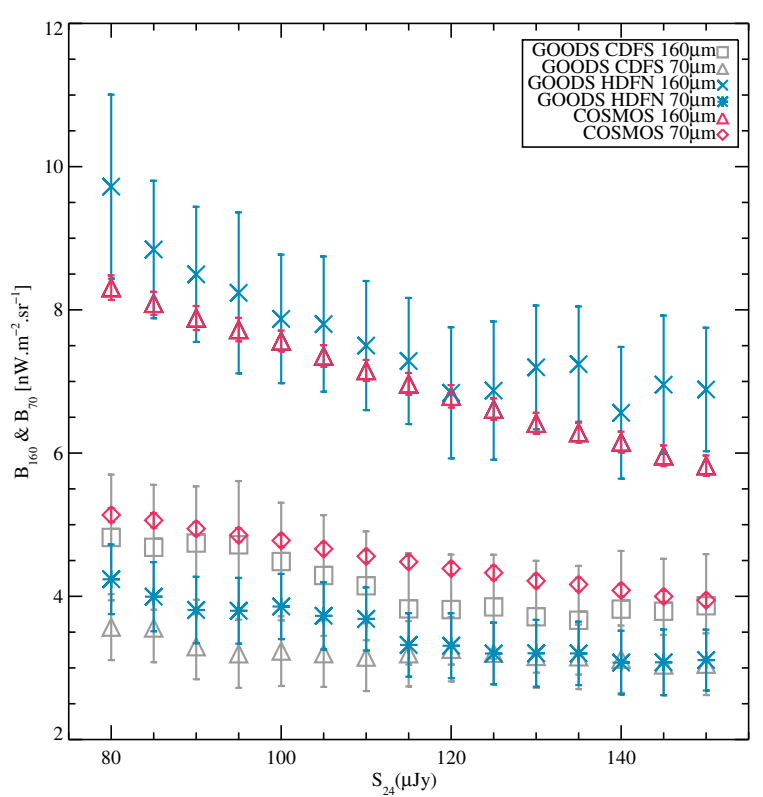

Fig. 2. Cumulative stacked brightness at $70 \mu \mathrm{m}$ and $160 \mu \mathrm{m}$ (in $\mathrm{nW} \mathrm{m} \mathrm{m}^{-2} \mathrm{sr}^{-1}$ ) on the CLEANed maps, as a function of the $24 \mu \mathrm{m}$ flux of our sample, regardless of the redshift. the 3 fields are represented at 160 and $70 \mu \mathrm{m}$ : COSMOS, GOODS-N (HDFN), and GOODS-S (CDFS).

(Dole et al. 2006; Bethermin et al. 2010b). This method consisted of stacking the 70 and $160 \mu \mathrm{m}$ maps at the location of the galaxies detected at $24 \mu \mathrm{m}$. The use of this method is justified for two reasons: 1) The $24 \mu \mathrm{m}$ population is a good proxy for the $70 \mu \mathrm{m}$ and $160 \mu \mathrm{m}$ populations making up most of the CIB near its peak (Dole et al. 2006; Bethermin et al. 2010a). 2) Only a few sources are individually detected at FIR wavelengths and do not resolve much of the background (Dole et al. 2004; Frayer et al. 2006a; Frayer et al. 2006b; Dole et al. 2006; Frayer et al. 2009). We note that stacking may be affected by galaxy clustering, since the stacked image shows two dimensions the two point angular correlation function (Dole et al. 2006; Bavouzet 2008; Bethermin et al. 2010a). However, with the Spitzer and Herschel beams, the effects of clustering on the stacking are not important (less than 15\%) (Bavouzet 2008; Fernandez-Conde et al. 2008, 2010).

We first stacked the $70 \mu \mathrm{m}$ and the $160 \mu \mathrm{m}$ MIPS data (CLEANed maps) as a function of the $24 \mu \mathrm{m}$ flux, regardless of the redshift of the sources (Fig. 2). This allowed us to check the consistency of the procedure, since the total brightness measured for stacks down to $S_{24}=80 \mu \mathrm{Jy}$ should be equal to the sum of the brightnesses obtained in redshift slices, as well as identify possible biases. The stacks in the COSMOS and the two GOODS fields at 70 and $160 \mu \mathrm{m}$ show strong dependencies on the fields: while COSMOS and GOODS-N (HDFN) stacks are consistent within $20 \%$, stacks in CDFS field have systematically fluxes lower than those of COSMOS by a factor of about 1.4-1.8. The higher quality statistics of the COSMOS field (surface area and number of sources) limits the impact of the variance caused by the large scale structure, and we attribute the systematically lower values of GOODS-S to this effect.

Prior to stacking the $24 \mu \mathrm{m}$ catalog into redshift bins on the $70 \mu \mathrm{m}$ and $160 \mu \mathrm{m}$ maps, we used the CLEAN algorithm (Hogbom 1974) to subtract the few resolved sources present in the far-infrared maps, to remove any bias in the resulting photometry of the stacked images. The stacking analysis presented on Fig. 2 was also done with the cleaned far-infrared maps. In the COSMOS field, we removed the sources brighter than $80 \mathrm{mJy}$ and $20 \mathrm{mJy}$ at $160 \mu \mathrm{m}$ and $70 \mu \mathrm{m}$ respectively, levels corresponding to 90 to $95 \%$ completeness and computed by Monte-Carlo simulations on the data themselves (Bethermin et al. 2010a). In both GOODS fields, we removed all the detected sources at $160 \mu \mathrm{m} \& 70 \mu \mathrm{m}$ identified at $24 \mu \mathrm{m}$, which corresponds to removing all sources brighter than $19 \mathrm{mJy}$ at $160 \mu \mathrm{m}$ ( 5 sources in GOODS HDFN \& 12 sources in GOODS CDFS) and $4.4 \mathrm{mJy}$ at $70 \mu \mathrm{m}$ ( 8 sources in GOODS HDFN \& 17 sources in GOODS CDFS). These brightest source detections at 70 and $160 \mu \mathrm{m}$ were individually identified at $24 \mu \mathrm{m}$ without ambiguity, and the redshift of the $24 \mu \mathrm{m}$ source is used. The flux densities of the removed detected sources were converted into brightnesses, and were added at the very end of the process to account for their CIB contribution (even if only a small fraction at far-IR wavelengths).

We estimated the AGN contribution to the CIB as a function of redshift using the identifications described in Sects. 2.1 and 2.2.

The stacking procedure wass performed for each redshift bin independently, and the images of the stacks are presented in Figs. 3 and 4, for the GOODS and COSMOS fields, respectively, together with the measured signal-to-noise ratios (hereafter $\mathrm{S} / \mathrm{Ns}$ ).

In our approach, we:

- compute the brightness (in redshift bins) of the detected sources that are removed from the maps to create the cleaned maps;

- select galaxies at $24 \mu \mathrm{m}$ (either all of them, only AGN, or only non-AGN) by redshift bins;

- stack at the positions of the selected galaxies in the 70 and $160 \mu$ m cleaned maps;

- perform photometry and bootstrap on those stacks;

- compute the uncertainty budget.

All measurements discussed in this section, i.e. number of stacked sources, resolved sources, AGN, and resulting brightnesses as a function of redshift, are summarized in Tables 2-5. 


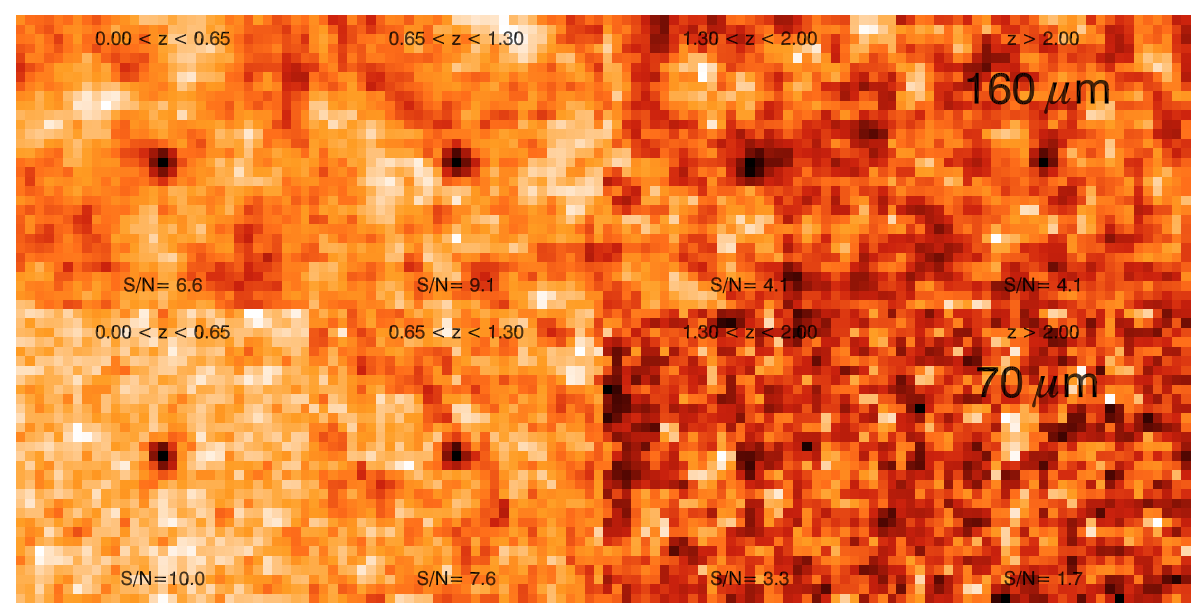

Fig. 3. Images of all the stacked galaxies in the $160 \mu \mathrm{m}$ (top) and $70 \mu \mathrm{m}$ (bottom) GOODS CLEANed maps by redshift bin (right to left): $0<z<0.65,0.65<z<1.3,1.3<z<2$, and $z>2$. The signal-to-noise ratio $(\mathrm{S} / \mathrm{N})$ is indicated in each image. Note the detection in the two first redshift bins at both wavelengths. Images are $305 \times 305$ sq. arcsec. wide at $70 \mu \mathrm{m}$ (with 9.85 arcsec pixel plate), and $496 \times 496 \mathrm{sq}$. arcsec. at $160 \mu \mathrm{m}$ (with 16 arcsec pixel plate). The PSF FWHM being 18 arcsec and $40 \operatorname{arcsec}$ at $70 \mu \mathrm{m}$ and $160 \mu \mathrm{m}$ respectivly, the PSF shown on these figures has about the same extent of 1.8 to 2.5 pixels at both wavelengths.

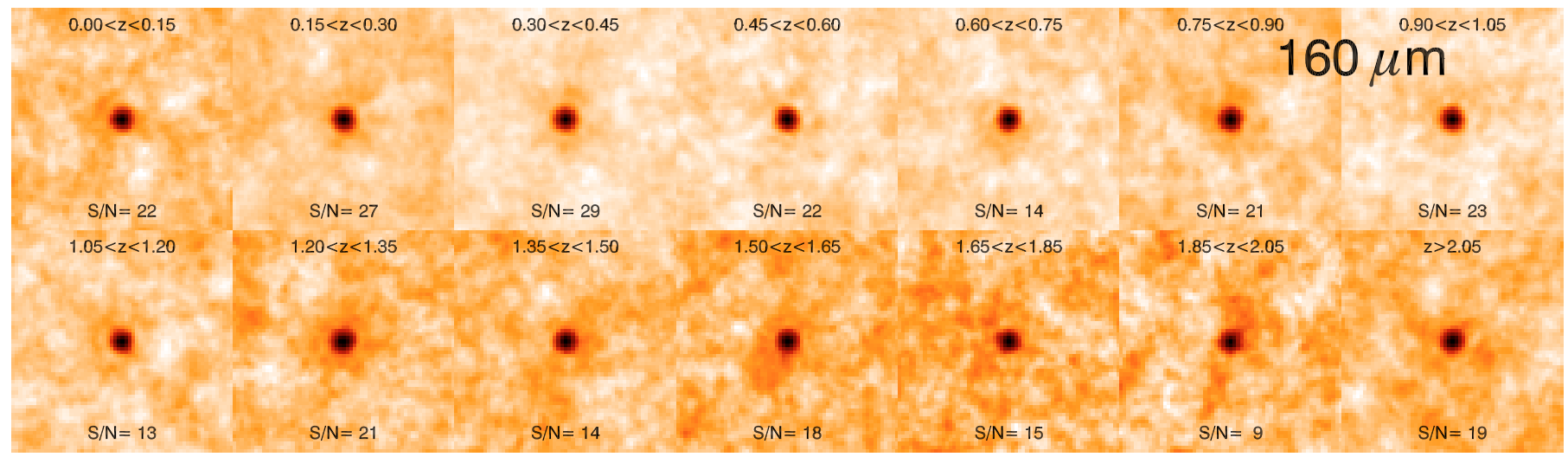

Fig. 4. Images of all the stacked galaxies in the $160 \mu \mathrm{m}$ COSMOS CLEANed maps with 14 redshift bins (left to right and top to bottom). The $\mathrm{S} / \mathrm{N}$ is indicated in each image. Note the clear detections in all redshift bins at both wavelengths. Images are $488 \times 488 \mathrm{sq}$. arcsec at $160 \mu \mathrm{m}$ (with 8 arcsec pixel plate). The PSF $F W H M$ of 40 arcsec corresponds to 5 pixels in these images.

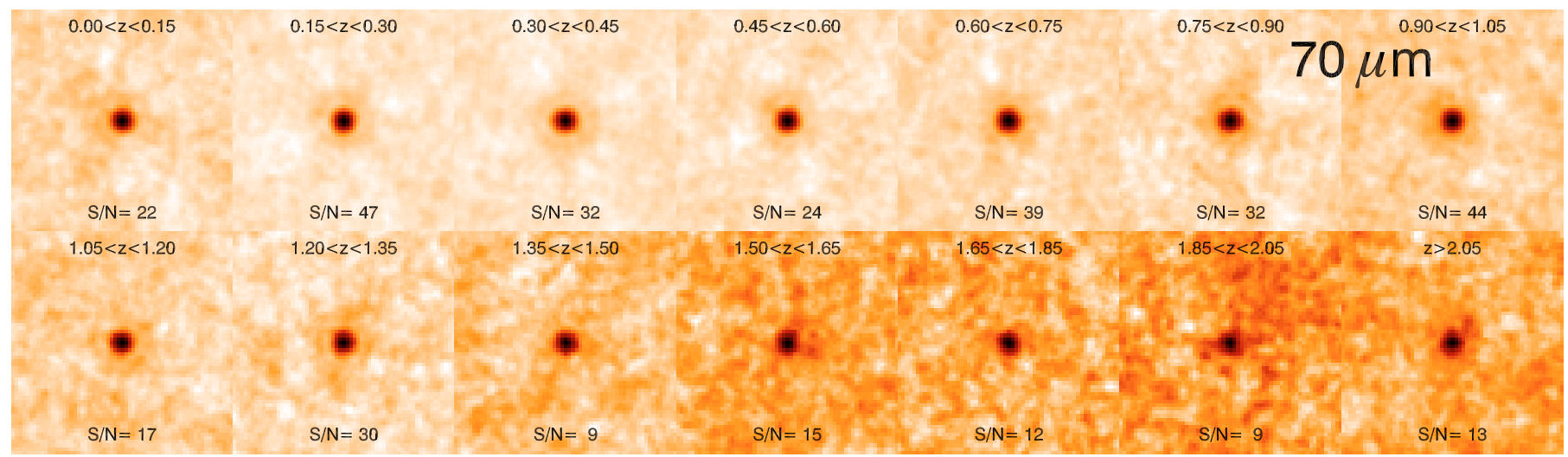

Fig. 5. Images of all the stacked galaxies in the $70 \mu \mathrm{m}$ COSMOS CLEANed maps with 14 redshift bins. Images are $244 \times 244$ sq. arcsec wide at $70 \mu \mathrm{m}$ (with 4 arcsec pixel plate). The PSF FWHM of 18 arcsec corresponds to 4.5 pixels in these images.

\subsection{Photometry and uncertainty estimations}

We performed aperture photometry on the stacked images with the following parameters at $160 \mu \mathrm{m}$ : aperture radius of 25 arcsec, a sky annulus to estimate the background between 80 arcsec and $110 \mathrm{arcsec}$, and an aperture correction of 2.29. At $70 \mu \mathrm{m}$, the respective parameters were: 18 arcsec, 50 arcsec and 70 arcsec, and 1.68. We have secure detections in all redshift bins at $160 \mu \mathrm{m}$ and $70 \mu \mathrm{m}$, except in the two highest redshift bin $(z>1.3)$ in GOODS. The signal-to-noise ratio (hereafter $\mathrm{S} / \mathrm{N}$ ) is higher for COSMOS data than that of GOODS, because of the larger number of sources used (the number of sources used are reported in 
M. Jauzac et al.: The CIB buildup since $z=2$ at 70 and $160 \mu \mathrm{m}$ in COSMOS and GOODS.

Table 4. The CIB brightness by redshift range at $160 \mu \mathrm{m}$, in units of $\mathrm{nW} \mathrm{m} \mathrm{m}^{-2} \mathrm{sr}^{-1}$, in the case of the COSMOS field.

\begin{tabular}{|c|c|c|c|c|c|c|c|}
\hline & $0<z<0.15$ & $0.15<z<0.3$ & $0.3<z<0.45$ & $0.45<z<0.6$ & $0.6<z<0.75$ & $0.75<z<0.9$ & $0.9<z<1.05$ \\
\hline$N_{\text {stack }}$ & 2083 & 1559 & 2853 & 2201 & 3225 & 3590 & 3478 \\
\hline$N_{\text {sources }}$ & 56 & 40 & 18 & 9 & 11 & 12 & 6 \\
\hline$N_{\mathrm{AGNs}}$ & 34 & 32 & 74 & 48 & 88 & 109 & 123 \\
\hline$B_{160_{\text {stack }}}$ & $0.43 \pm 0.04$ & $0.56 \pm 0.04$ & $0.87 \pm 0.05$ & $0.63 \pm 0.04$ & $0.76 \pm 0.07$ & $0.75 \pm 0.06$ & $0.71 \pm 0.06$ \\
\hline$B_{160_{\text {sources }}}$ & $0.3 \pm 0.001$ & $0.21 \pm 0.002$ & $0.07 \pm 0.001$ & $0.03 \pm 0.001$ & $0.034 \pm 0.001$ & $0.043 \pm 0.001$ & $0.021 \pm 0.001$ \\
\hline$B_{160_{\mathrm{AGNS}}}$ & $0.02 \pm 0.006$ & $0.01 \pm 0.005$ & $0.03 \pm 0.01$ & $0.02 \pm 0.01$ & $0.02 \pm 0.01$ & $0.01 \pm 0.01$ & $0.02 \pm 0.01$ \\
\hline \multirow[t]{2}{*}{$B_{160_{\text {tot }}}$} & $0.72 \pm 0.04$ & $0.77 \pm 0.04$ & $0.93 \pm 0.05$ & $0.66 \pm 0.04$ & $0.8 \pm 0.07$ & $0.8 \pm 0.06$ & $0.73 \pm 0.06$ \\
\hline & $1.05<z<1.2$ & $1.2<z<1.35$ & $1.35<z<1.5$ & $1.5<z<1.65$ & $1.65<z<1.85$ & $1.85<z<2.05$ & $z>2.05$ \\
\hline$N_{\text {stack }}$ & 2670 & 1401 & 2044 & 1311 & 1519 & 2073 & 2833 \\
\hline$N_{\text {sources }}$ & 2 & 0 & 2 & 2 & 0 & 1 & 2 \\
\hline$N_{\mathrm{AGNs}}$ & 83 & 76 & 55 & 225 & 230 & 198 & 288 \\
\hline$B_{160_{\text {stack }}}$ & $0.52 \pm 0.05$ & $0.33 \pm 0.09$ & $0.36 \pm 0.04$ & $0.26 \pm 0.03$ & $0.22 \pm 0.02$ & $0.27 \pm 0.04$ & $0.47 \pm 0.04$ \\
\hline$B_{160_{\text {sources }}}$ & $0.005 \pm 0.0003$ & - & $0.006 \pm 0.0003$ & $0.006 \pm 0.0003$ & - & $0.003 \pm 0.0001$ & $0.007 \pm 0.0003$ \\
\hline$B_{160_{\mathrm{AGNS}}}$ & $0.02 \pm 0.01$ & $0.02 \pm 0.01$ & $0.01 \pm 0.008$ & $0.04 \pm 0.03$ & $0.04 \pm 0.03$ & $0.05 \pm 0.03$ & $0.04 \pm 0.03$ \\
\hline$B_{160_{\text {tot }}}$ & $0.53 \pm 0.05$ & $0.33 \pm 0.09$ & $0.37 \pm 0.04$ & $0.27 \pm 0.03$ & $0.22 \pm 0.02$ & $0.28 \pm 0.04$ & $0.47 \pm 0.04$ \\
\hline
\end{tabular}

Notes. The subscript "stack" refers to the measurement of the signal on the cleaned and stacked image; "sources" to the individually detected sources; "AGN" to the sources identified as AGN by Salvato et al. (2009). The total number of sources used in this analysis is thus $N_{\text {stack }}+N_{\text {sources }}$.

Table 5. The CIB brightness by redshift range at $70 \mu \mathrm{m}$, in units of $\mathrm{nW} \mathrm{m}^{-2} \mathrm{sr}^{-1}$, in the COSMOS field.

\begin{tabular}{lccccccc}
\hline \hline & $0<z<0.15$ & $0.15<z<0.3$ & $0.3<z<0.45$ & $0.45<z<0.6$ & $0.6<z<0.75$ & $0.75<z<0.9$ & $0.9<z<1.05$ \\
\hline$N_{\text {stack }}$ & 2083 & 1559 & 2853 & 2202 & 3225 & 3590 & 3478 \\
$N_{\text {sources }}$ & 77 & 82 & 48 & 23 & 13 & 11 & 3 \\
$N_{\text {AGNs }}$ & 34 & 32 & 74 & 48 & 88 & 110 & 123 \\
\hline$B_{70_{\text {stack }}}$ & $0.31 \pm 0.03$ & $0.39 \pm 0.02$ & $0.6 \pm 0.03$ & $0.42 \pm 0.03$ & $0.47 \pm 0.03$ & $0.41 \pm 0.03$ & $0.39 \pm 0.03$ \\
$B_{70_{\text {sources }}}$ & $0.31 \pm 0.001$ & $0.34 \pm 0.001$ & $0.13 \pm 0.0005$ & $0.05 \pm 0.0003$ & $0.04 \pm 0.0003$ & $0.027 \pm 0.0003$ & $0.008 \pm 0.0001$ \\
$B_{70_{\text {AGNs }}}$ & $0.011 \pm 0.004$ & $0.008 \pm 0.003$ & $0.015 \pm 0.006$ & $0.009 \pm 0.003$ & $0.016 \pm 0.005$ & $0.015 \pm 0.007$ & $0.015 \pm 0.008$ \\
$B_{70_{\text {tot }}}$ & $0.62 \pm 0.03$ & $0.73 \pm 0.02$ & $0.73 \pm 0.03$ & $0.47 \pm 0.03$ & $0.51 \pm 0.03$ & $0.44 \pm 0.03$ & $0.4 \pm 0.03$ \\
\hline & $1.05<z<1.2$ & $1.2<z<1.35$ & $1.35<z<1.5$ & $1.5<z<1.65$ & $1.65<z<1.85$ & $1.85<z<2.05$ & $z>2.05$ \\
\hline$N_{\text {stack }}$ & 2670 & 1401 & 2044 & 1311 & 1519 & 2073 & 0 \\
$N_{\text {sources }}$ & 2 & 1 & 2 & 0 & 0 & 2833 \\
$N_{\text {AGNs }}$ & 83 & 76 & 55 & 225 & 232 & 0 \\
\hline$B_{70_{\text {stack }}}$ & $0.25 \pm 0.02$ & $0.18 \pm 0.01$ & $0.17 \pm 0.02$ & $0.1 \pm 0.01$ & $0.09 \pm 0.02$ & $0.09 \pm 0.02$ & $0.16 \pm 0.02$ \\
$B_{70_{\text {sources }}}$ & $0.004 \pm 0.0001$ & $0.002 \pm 0.0001$ & $0.003 \pm 0.0001$ & - & - & - & - \\
$B_{70_{\text {AGNs }}}$ & $0.14 \pm 0.007$ & $0.011 \pm 0.005$ & $0.006 \pm 0.004$ & $0.28 \pm 0.012$ & $0.026 \pm 0.015$ & $0.022 \pm 0.01$ & $0.029 \pm 0.018$ \\
$B_{70_{\text {tot }}}$ & $0.26 \pm 0.02$ & $0.18 \pm 0.01$ & $0.17 \pm 0.02$ & $0.1 \pm 0.01$ & $0.09 \pm 0.2$ & $0.09 \pm 0.02$ & $0.15 \pm 0.02$ \\
\hline
\end{tabular}

Notes. Terms are defined in the caption of Table 4.

Table 2. The CIB brightness by redshift range at $160 \mu \mathrm{m}$, in units of $\mathrm{nW} \mathrm{m}{ }^{-2} \mathrm{sr}^{-1}$, in the case of the GOODS fields.

\begin{tabular}{lcccc}
\hline \hline & $0<z<0.65$ & $0.65<z<1.3$ & $1.3<z<2$ & $z>2$ \\
\hline$N_{\text {stack }}$ & 317 & 573 & 258 & 198 \\
$N_{\text {sources }}$ & 10 & 11 & 3 & 2 \\
$N_{\text {AGNs }}$ & 9 & 45 & 38 & 44 \\
\hline$B_{160_{\text {stack }}}$ & $2.18 \pm 0.45$ & $2.39 \pm 0.54$ & $1.13 \pm 0.35$ & $0.78 \pm 0.27$ \\
$B_{160_{\text {sources }}}$ & $0.57 \pm 0.18$ & $0.34 \pm 0.11$ & $0.06 \pm 0.04$ & $0.07 \pm 0.05$ \\
$B_{160_{\text {AGN }}}$ & $0.04 \pm 0.07$ & $0.17 \pm 0.25$ & $0.32 \pm 0.22$ & $0.20 \pm 0.19$ \\
$B_{160_{\text {tot }}}$ & $2.75 \pm 0.46$ & $2.73 \pm 0.54$ & $1.19 \pm 0.35$ & $0.85 \pm 0.27$ \\
\hline
\end{tabular}

Notes. The subscript "stack" refers to the measurement of the signal on the cleaned and stacked image; "sources" to the individually detected sources; "AGN" to the sources identified as AGN by Caputi et al. (2006). The total number of sources used in this analysis is thus $N_{\text {stack }}+N_{\text {sources }}$.
Table 3. The CIB brightness by redshift range at $70 \mu \mathrm{m}$, in units of $\mathrm{nW} \mathrm{m}{ }^{-2} \mathrm{sr}^{-1}$, in the GOODS fields.

\begin{tabular}{lcccc}
\hline \hline & $0<z<0.65$ & $0.65<z<1.3$ & $1.3<z<2$ & $z>2$ \\
\hline$N_{\text {stack }}$ & 317 & 575 & 259 & 198 \\
$N_{\text {sources }}$ & 19 & 5 & 1 & 0 \\
$N_{\text {AGNs }}$ & 9 & 45 & 38 & 44 \\
\hline$B_{70_{\text {stack }}}$ & $1.29 \pm 0.22$ & $1.25 \pm 0.28$ & $0.35 \pm 0.15$ & $0.15 \pm 0.12$ \\
$B_{70_{\text {sources }}}$ & $0.77 \pm 0.18$ & $0.14 \pm 0.07$ & $0.01 \pm 0.015$ & - \\
$B_{70_{\text {AGNs }}}$ & $0.05 \pm 0.03$ & $0.1 \pm 0.12$ & $0.11 \pm 0.08$ & $0.11 \pm 0.12$ \\
$B_{70_{\text {tot }}}$ & $2.06 \pm 0.24$ & $1.4 \pm 0.28$ & $0.36 \pm 0.15$ & $0.15 \pm 0.12$ \\
\hline
\end{tabular}

Notes. Terms are defined in the caption of Table 2.

Tables 2 to 5). In our analysis, we thus considered the redshifts bins $0<z<1.3$ in GOODS and $0<z<2.5$ in COSMOS. 

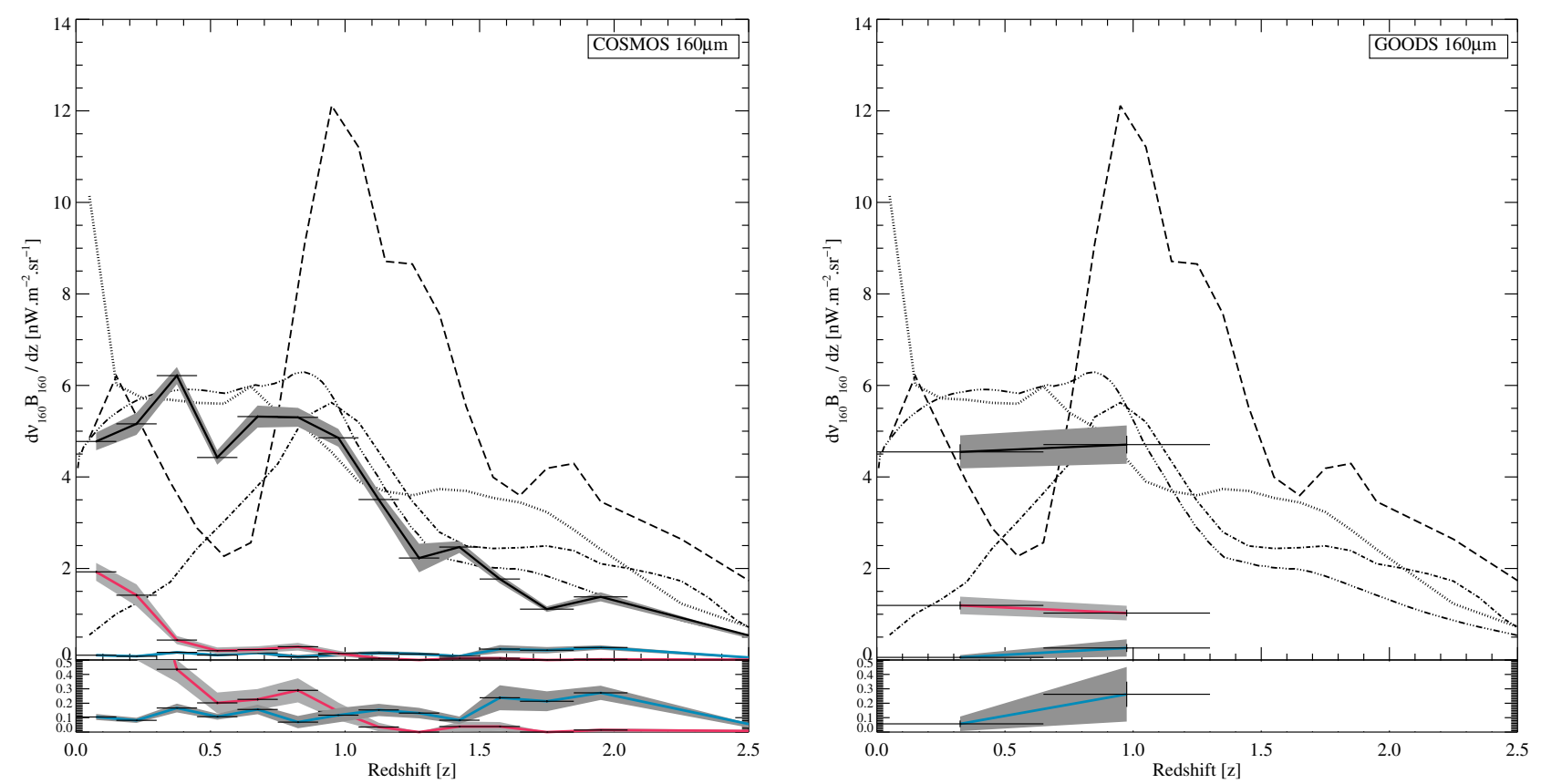

Fig. 6. Differential $160 \mu \mathrm{m}$ background brightness $\mathrm{d} v_{160} B_{160} / \mathrm{d} z$ as a function of redshift, in units of $\mathrm{nW} \mathrm{m}^{-2} \mathrm{sr}^{-1}$, in the COSMOS field $(l e f t)$ and GOODS field (right). The lower part of the plot shows a linear zoom between 0 and $0.5 \mathrm{nW} \mathrm{m}^{-2} \mathrm{sr}^{-1}$ to show more clearly the trend of cleaned sources and AGNs. Black solid lines: total contribution of infrared galaxies. Solid red lines: contribution from resolved sources only. Solid blue line: contribution from AGN only. Dashed line: Lagache et al. (2004) model, with $S_{24}>80 \mu$ Jy cut. Dotted line: Le Borgne et al. (2009) model, with $S_{24}>80 \mu$ Jy cut. Dot-dash line: Valiante et al. (2009). Dot-dot-dot-dash line: Bethermin et al. (2010c) model, with $S_{24}>80 \mu$ Jy cut. Models are presented and discussed in Sect. 4.5 and Fig. 10.

The error bars correspond to three quadratically summed terms: (1-) the photometry uncertainty, (2-) the Poisson noise coming from the number of stacked sources, and (3-) a bootstrap analysis.

The bootstrap analysis involved repeating the stacking process $N_{\mathrm{b}}$ times (usually $N_{\mathrm{b}}=5000$ and $N_{\mathrm{b}}=14000$ for GOODS and COSMOS data respectively) of a new sample composed of randomly selected sources from our original sample, keeping the total number of sources constant (Bavouzet 2008); this means that some stacked positions might be present zero, or multiple times in each realization. The bootstrap error bars correspond to the standard deviation in the distribution of the photometry measured for these $N_{\mathrm{b}}$ realizations. We note that the $\mathrm{S} / \mathrm{N}$ of the detections in the stacked images (only photometric) is higher than the value quoted in this paper, since we added the Poisson and bootstrap terms to estimate the final error bar, which takes into account the dispersion in the underlying sample. The final error bar is thus larger than just the photometric noise estimate. The error bars for the AGN samples were determined using a smaller number of bootstrap iterations, $N_{\mathrm{b}}=100$ and $N_{\mathrm{b}}=2000$ for GOODS and COSMOS, respectively.

The variance due to large-scale structure (also known as cosmic variance) and field-to-field variations are a systematic component of the noise, which is difficult to estimate at this stage. The Poisson noise, used here, provides a strict lower limit of the cosmic variance.

\subsection{Measurements}

By adding the brightnesses derived by the stacking analysis of $24 \mu \mathrm{m}$ sources with $S_{24} \geq 80 \mu \mathrm{Jy}$ and by the few detected far-infrared sources, we measured
$B_{160_{\text {tot }-\mathrm{CoOps}}}=7.53 \pm 0.52 \mathrm{nW} \mathrm{m}^{-2} \mathrm{sr}^{-1}$ at $160 \mu \mathrm{m}$, $B_{70_{\text {tot-GOODS }}}=3.97 \pm 0.17 \mathrm{nW} \mathrm{m}^{-2} \mathrm{sr}^{-1}$ at $70 \mu \mathrm{m}$ and $B_{160_{\text {tot-Cosmos }}}=7.88 \pm 0.19 \mathrm{nW} \mathrm{m}^{-2} \mathrm{sr}^{-1}$ at $160 \mu \mathrm{m}$, $B_{70_{\text {tot-Cosmos }}}=4.95 \pm 0.08 \mathrm{nW} \mathrm{m}^{-2} \mathrm{sr}^{-1}$ at $70 \mu \mathrm{m}$ (see also the summary in Table 6).

If we compare these brightnesses with the models from Lagache et al. (2004), Le Borgne et al. (2009), and Bethermin et al. (2010c) (cf. Sect. 4.5 and Table 6) applying the same selection of using the $24 \mu \mathrm{m}$ sources with $S_{24} \geq 80 \mu \mathrm{Jy}$, we find that we can resolve between $66 \%$ and $89 \%$ of the $160 \mu \mathrm{m}$ background, and between $75 \%$ and $98 \%$ of the $70 \mu \mathrm{m}$ background.

Using only the post-Herschel model in hand (Bethermin et al. 2010c), our data indicate that we can resolve in COSMOS data $90 \%$ at $160 \mu \mathrm{m}$ and $98 \%$ at $70 \mu \mathrm{m}$ of the background we should detect by applying the selection at $24 \mu \mathrm{m}$. Our selection introduces an incompleteness in the CIB estimate corresponding to the fainter $24 \mu \mathrm{m}$ sources $\left(S_{24}<80 \mu \mathrm{Jy}\right)$, omitted from our analysis; this omission implies that we resolve $68 \%$ of the total $160 \mu \mathrm{m}$ background and $81 \%$ of the total $70 \mu \mathrm{m}$ background in COSMOS (see Sect. 4.5 for the details). For comparison, Berta et al. (2010) identified about 50\% of the $100 \mu \mathrm{m}$ and $160 \mu \mathrm{m}$ backgrounds with individual sources, and account between $50 \%$ and $75 \%$ of the background when stacking at the positions of $24 \mu \mathrm{m}$ galaxies, as we do.

\section{Discussion}

We now present both the measurements and models in the form of $\frac{\mathrm{d}\left(v B_{v}\right)}{\mathrm{d} z}$ versus redshift $z$, where $v B_{v}$ is the CIB brightness in $\mathrm{nW} \mathrm{m}{ }^{-2} \mathrm{sr}^{-1}, \lambda$ is the wavelength (70 $\mu \mathrm{m}$ or $\left.160 \mu \mathrm{m}\right)$, and $v$ is the corresponding frequency. This representation has the advantage of being independent of the redshift binning, thus allowing 

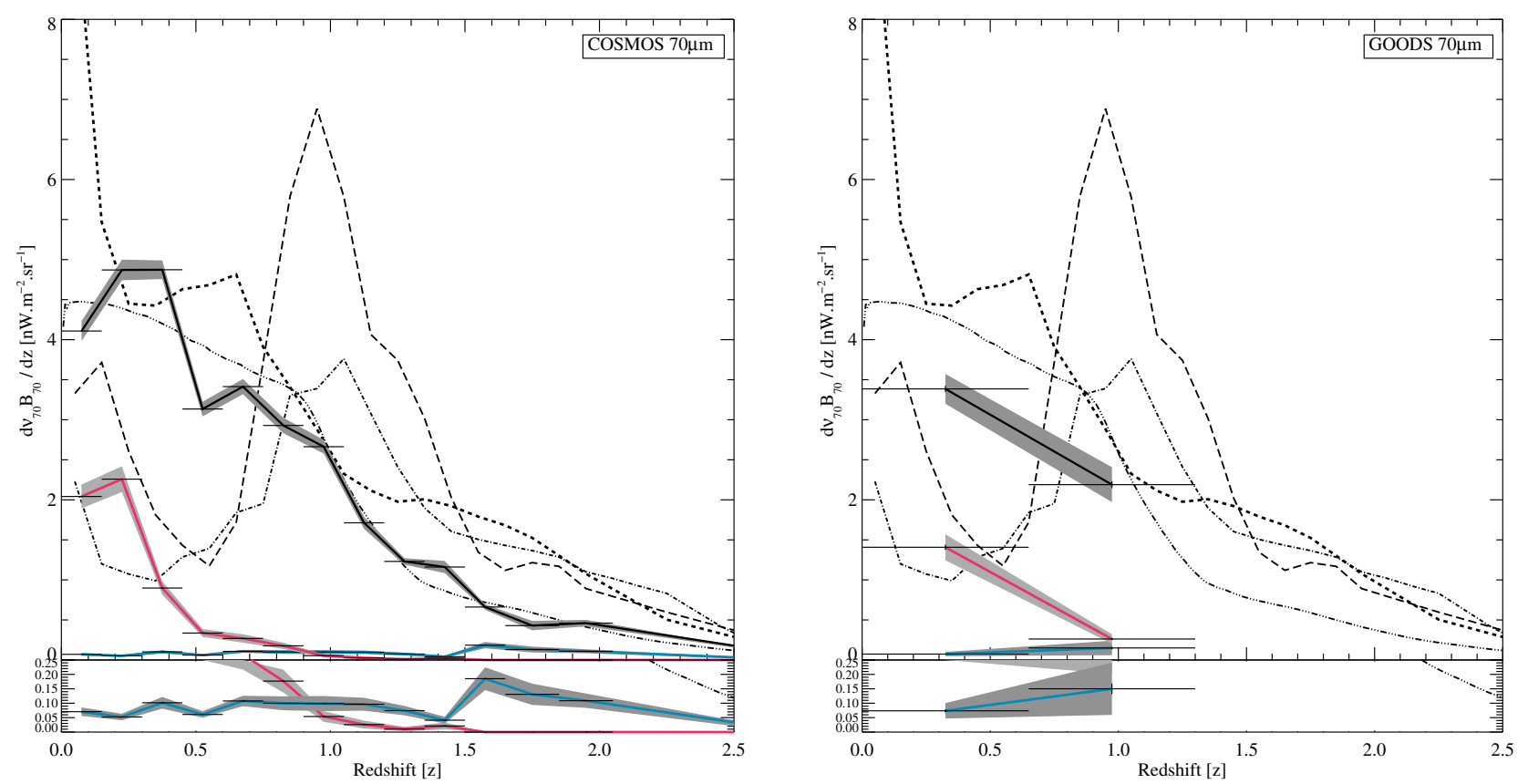

Fig. 7. Differential $70 \mu \mathrm{m}$ background brightness $\mathrm{d} v_{70} B_{70} / \mathrm{d} z$ as a function of redshift, in units of $\mathrm{nW} \mathrm{m}^{-2} \mathrm{sr}^{-1}$. in the COSMOS field (left) and GOODS field (right). The lower part of the plot shows a linear zoom between 0 and $0.25 \mathrm{nW} \mathrm{m}^{-2} \mathrm{sr}^{-1}$ to show more clearly the trend of cleaned sources and AGNs. Black solid lines: total contribution of infrared galaxies. Solid red lines: contribution from resolved sources only. Solid blue line: contribution from AGN only. Dashed line: Lagache et al. (2004) model, with $S_{24}>80 \mu$ Jy cut. Dotted line: Le Borgne et al. (2009) model, with $S_{24}>80 \mu$ Jy cut. Dot-dash line: Valiante et al. (2009). Dot-dot-dot-dash line: Bethermin et al. (2010c) model, with $S_{24}>80 \mu$ Jy cut. Models are presented and discussed in Sect. 4.5 and Fig. 10.

a direct comparison between datasets and models sampled in different ways into redshift bins. We discuss data and models with the prior selection of $S_{24}>80 \mu \mathrm{Jy}$, and show (Sect. 4.5) that our conclusions for $z<1.5$, i.e., where most of the FIR background originates, are no different with this prior selection than when including fainter galaxies.

\subsection{The $160 \mu \mathrm{m}$ background: its history since $z=2$}

The distribution of the $160 \mu \mathrm{m}$ CIB measured brightness as a function of redshift (Fig. 6) shows a plateau between redshifts 0.3 and 0.9 in both the COSMOS and GOODS fields, followed by a decrease at higher redshift. The small dip at $z=0.5$ in COSMOS is not significant, since it disappears when the size of the redshift bin is increased $(\Delta z=0.3$ instead of 0.15$)$ and is probably caused by a structure in the COSMOS field. The GOODS field exhibits the same trend in redshift.

The contribution from resolved sources reaches a maximum at $z<0.3$ and strongly decreases toward higher redshift, in agreement with the identifications of Frayer et al. (2006a). The AGN contribution is rather constant with redshift; the relative contribution of AGN thus increases with redshift. Assuming that the COSMOS field is representative of the whole CIB population, we find that $33 \%$ of the $160 \mu \mathrm{m}$ background is accounted for at redshifts $0<z<0.5,41 \%$ for $0.5<z<1$, $17 \%$ for $1<z<1.5$, and $9 \%$ for $1.5<z<2$. Our results are consistent with Berta et al. (2010), who analyzed a deep sample in the GOODS-N field at $160 \mu \mathrm{m}$ with PACS/Herschel. Most of the far-infrared sources are resolved by Herschel, and the stacks of $24 \mu \mathrm{m}$ sources provide slightly more depth. Their peak at $z=1$ is more pronounced than in our analysis.

The Lagache et al. (2004), Le Borgne et al. (2009), Valiante et al. (2009), and Bethermin et al. (2010c) models are
Table 6. The total CIB brightness at $160 \mu \mathrm{m} \& 70 \mu \mathrm{m}$ for the GOODS $\&$ COSMOS fields, in units of $\mathrm{nW} \mathrm{m}^{-2} \mathrm{sr}^{-1}$.

\begin{tabular}{lcc}
\hline \hline & $160 \mu \mathrm{m}$ & $70 \mu \mathrm{m}$ \\
\hline$B_{\text {tot-GOODS }}$ & $7.53 \pm 0.84$ & $3.97 \pm 0.41$ \\
$B_{\text {tot-COSMOS }}$ & $7.88 \pm 0.19$ & $4.95 \pm 0.08$ \\
$B_{\text {Bethermin }}$ & $9.0 \pm 1.1$ & $5.2 \pm 0.4$ \\
$B_{\text {Bethermin }_{\text {CIBestimate }}}$ & $14.6_{-2.9}^{+7.1}$ & $6.4_{-0.6}^{+0.7}$ \\
$B_{\text {model }_{\text {Lagache }}}\left(S_{24}>80 \mu \mathrm{Jy}\right)$ & 11.91 & 5.73 \\
$B_{\text {model }_{\text {Lagache }}}$ & 14.87 & 6.78 \\
$B_{\text {model }_{\text {LeBorgne }}}\left(S_{24}>80 \mu \mathrm{Jy}\right)$ & 9.54 & 6.65 \\
$B_{\text {model }_{\text {LeBorgne }}}$ & 13.57 & 8.54 \\
$B_{\text {model }_{\text {Valiante }}}\left(S_{24}>80 \mu \mathrm{Jy}\right)$ & 6.84 & 4.27 \\
$B_{\text {model }_{\text {Valiante }}}\left(S_{24}>80 \mu \mathrm{Jy}\right)$ & 16.70 & 6.98 \\
$B_{\text {model }_{\text {Bethermin }}}$ & 8.82 & 5.02 \\
$B_{\text {model }_{\text {Bethermin }}}$ & 11.66 & 6.09 \\
\hline
\end{tabular}

Notes. Lines 1 \& 2: our estimates; line 3: Bethermin et al. (2010a) CIB measured value using number counts integration; line 4: Bethermin et al. (2010a) CIB value with extrapolation of the number counts in power-law; line 5: Lagache et al. (2004) CIB model value with the constraint: $S_{24}>80 \mu \mathrm{Jy}$; line 6: Lagache et al. (2004) CIB model value of the total background; line 7: Le Borgne et al. (2009) CIB model value with the constraint: $S_{24}>80 \mu \mathrm{Jy}$; line 8: Le Borgne et al. (2009) CIB model value of the total background; line 9: Valiante et al. (2009) CIB model value with the constraint: $S_{24}>80 \mu \mathrm{Jy}$; line 10: Valiante et al. (2009) CIB model value of the total background; line 11: Bethermin et al. (2010c) CIB model value with the constraint: $S_{24}>80 \mu \mathrm{Jy}$; line 12: Bethermin et al. (2010c) CIB model value of the total background.

overplotted to our measurements on Fig. 6, after applying the same selection of $S_{24} \geq 80 \mu \mathrm{Jy}$ as applied on the data. Pre-Herschel models predict different redshift distributions for 
$\mathrm{d} v_{160} B_{160} / \mathrm{d} z$. The Lagache et al. (2004) models peaks at $z \sim 1$ and desagrees with our data. Our data are in closer qualitative agreement with the Le Borgne et al. (2009) model (except for $z<0.3$ ), but no model is capable of reproducing the $z>1$ tail. The problem of the discrepancy between our data and the Le Borgne et al. (2009) model at $z<0.3$ might be twofold: our data is of lower statistical quality at very low redshift due to the relatively small sky area covered, and the model might be overpredicting low- $z$ galaxies because of the lack of a cold component in the galaxy SED used. The model of Bethermin et al. (2010c) provides a close fit to our data in the low, intermediate, and high redshift ranges, most likely because it is based on a optimal minimization between the model and the most recent Spitzer and Herschel data, and already takes into account the FIR and submm statistical properties of galaxies (see Sect. 4.5).

\subsection{The $70 \mu \mathrm{m}$ background: its history since $z=2$}

The distribution of the measured $70 \mu \mathrm{m}$ CIB measured brightness as a function of redshift (Fig. 7) clearly indicates that the greatest contribution is from sources at $z<0.5$ in COSMOS, in agreement with the GOODS measurements. The peak contribution at $70 \mu \mathrm{m}$ occurs at lower redshift than at $160 \mu \mathrm{m}$, which is expected as a consequence of the K-correction (the effect of the redshifted shape of the galaxy spectra). This difference is also seen between $100 \mu \mathrm{m}$ and $160 \mu \mathrm{m}$ in the PACS/Herschel data by Berta et al. (2010). The dip at $z \sim 0.5$ is most likely caused by cosmic variance, because it is not seen in the GOODS field and disappears when we use broader redshift bins. This dip does not affect our conclusions about the $70 \mu \mathrm{m}$ background emission with redshift.

The contribution from resolved sources reaches a maximum at $0.15<z<0.3$ and strongly decreases at higher redshift, in agreement with the identifications of Frayer et al. (2006a). The AGN contribution remains almost constant with redshift. Assuming that the COSMOS field is representative of the whole CIB population, we conclude that $43 \%$ of the $70 \mu \mathrm{m}$ background is accounted for at redshifts $0<z<0.5,38 \%$ for $0.5<z<1$, $13 \%$ for $1<z<1.5$, and $5 \%$ for $1.5<z<2$. Those results agree with those of Pascale et al. (2009).

The Lagache et al. (2004) model predicts that the CIB at $70 \mu \mathrm{m}$ reaches a maximum at around $z=1$, which is not seen in the data presented here. The Le Borgne et al. (2009) model predicts a peak at lower redshift $(z \leq 0.5)$ and a strong contribution at $z \sim 0$, neither of which seen in the data; otherwise, the decrease at $z>0.5$ has a shape comparable to the data, despite there being a larger high-redshift tail. The post-Herschel Bethermin et al. (2010c) model closely agrees with the observed evolution in both COSMOS and GOODS (Fig. 7).

\subsection{Role of $A G N$}

The AGN contribution in each redshift bin is shown in Figs. 7 and 6, as the lower blue area. The estimate of this contribution can be considered as a "best effort" estimate, because of the difficulty in identifying the origin of the far-infrared emission (star formation or AGN). Our AGN identification relies on X-ray detections and IRAC colors (Caputi et al. 2006; Salvato et al. 2009), but the far-infrared emission is not necessarily physically linked to the AGN (Le Floc'h et al. 2007).

Our analysis indicates that the absolute contribution of AGN to the CIB at $70 \mu \mathrm{m}$ and $160 \mu \mathrm{m}$ is almost constant with redshift. Figure 8 shows the relative contribution to the CIB, which

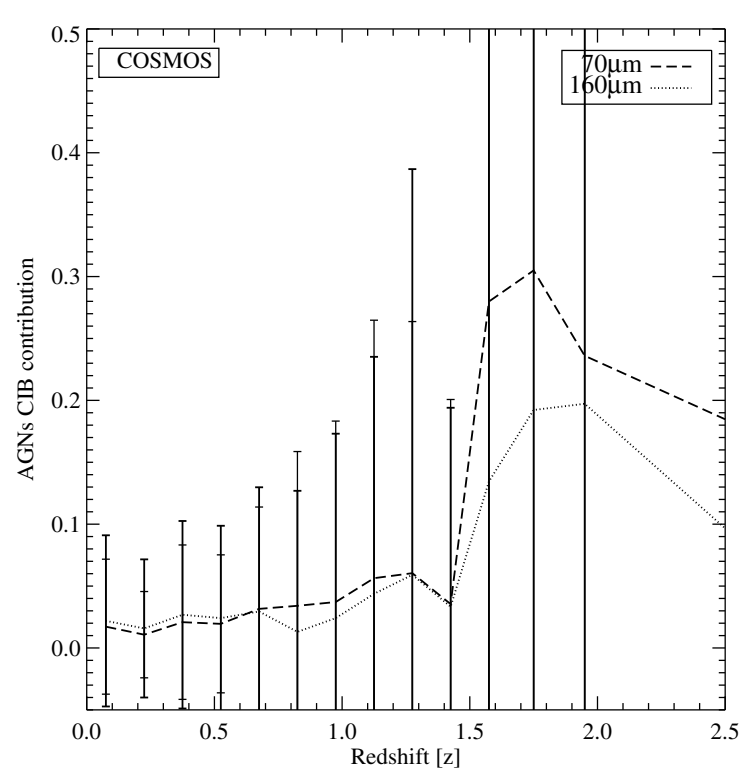

Fig. 8. The relative contribution of identified AGN to the $70 \mu \mathrm{m}$ and $160 \mu \mathrm{m} \mathrm{CIB}$ as a function of redshift, for the selection at $24 \mu \mathrm{m}$ given by $S_{24}>80 \mu \mathrm{Jy}$.

is obtained by dividing the AGN contribution by the total CIB contribution. Because of the smaller contribution of higher redshift sources to the CIB, the AGN fraction contribution to the CIB increases with redshift, from about $3 \pm 10 \%$ for $0<z<1.5$, and possibly up to $15-25 \%$ for $z>1.5$, but our large error bars do not allow any meaningful estimate. We thus can only state that the relative AGN contribution is smaller than about $10 \%$ at $z<1.5$. Our results agree with Daddi et al. (2007), who predict that the AGN contribution should not exceed about $7 \%$ up to a redshift of unity.

AGNs are understood to play a central role in terms of physical processes driving galaxy evolution and regulating star formation by means of feedback (e.g. Magorrian et al. 1998; Ferrarese \& Merritt 2000; Bower et al. 2006; Hopkins et al. 2006; Cattaneo et al. 2009; Hopkins et al. 2010). However, our work confirms that, in terms of total energy contributions to the CIB, the AGNs play a minor role. This conclusion agrees with the identifications of Frayer et al. (2006a) and the analysis of Valiante et al. (2009), which demonstrates (their Fig. 19) that fewer than $10 \%$ of the sources with $S_{24}>80 \mu$ Jy (i.e. the sources constituting the CIB at 24 to $160 \mu \mathrm{m}$, see Dole et al. 2006) are affected by a significant AGN contribution.

\subsection{The $24,70,100$, and $160 \mu \mathrm{m}$ backgrounds}

The mid- and far-infrared background buildup at 24, 70, 100, and $160 \mu \mathrm{m}$ as a function of redshift is presented in Fig. 9 and is available online ${ }^{3}$. The $24 \mu \mathrm{m}$ data from Le Floc'h et al. (2009) were normalized to $2.86 \mathrm{nW} \mathrm{m}^{-2} \mathrm{sr}^{-1}$ (Bethermin et al. 2010a), the data at $100 \mu \mathrm{m}$ are from Berta et al. (2010), but the data at 70 and $160 \mu \mathrm{m}$ come from this work. At wavelengths longer than $60 \mu \mathrm{m}$, the observed build-up sequence has an increasing contribution between $z \sim 0.5$ and $z \sim 1$ with increasing wavelength. This behavior is expected because of the redshifted peak in the galaxy spectral energy distributions, or k-correction (e.g. Lagache et al. 2004; Lagache et al. 2005). The $24 \mu \mathrm{m}$ distribution

3 http://www.ias.u-psud.fr/irgalaxies/ 


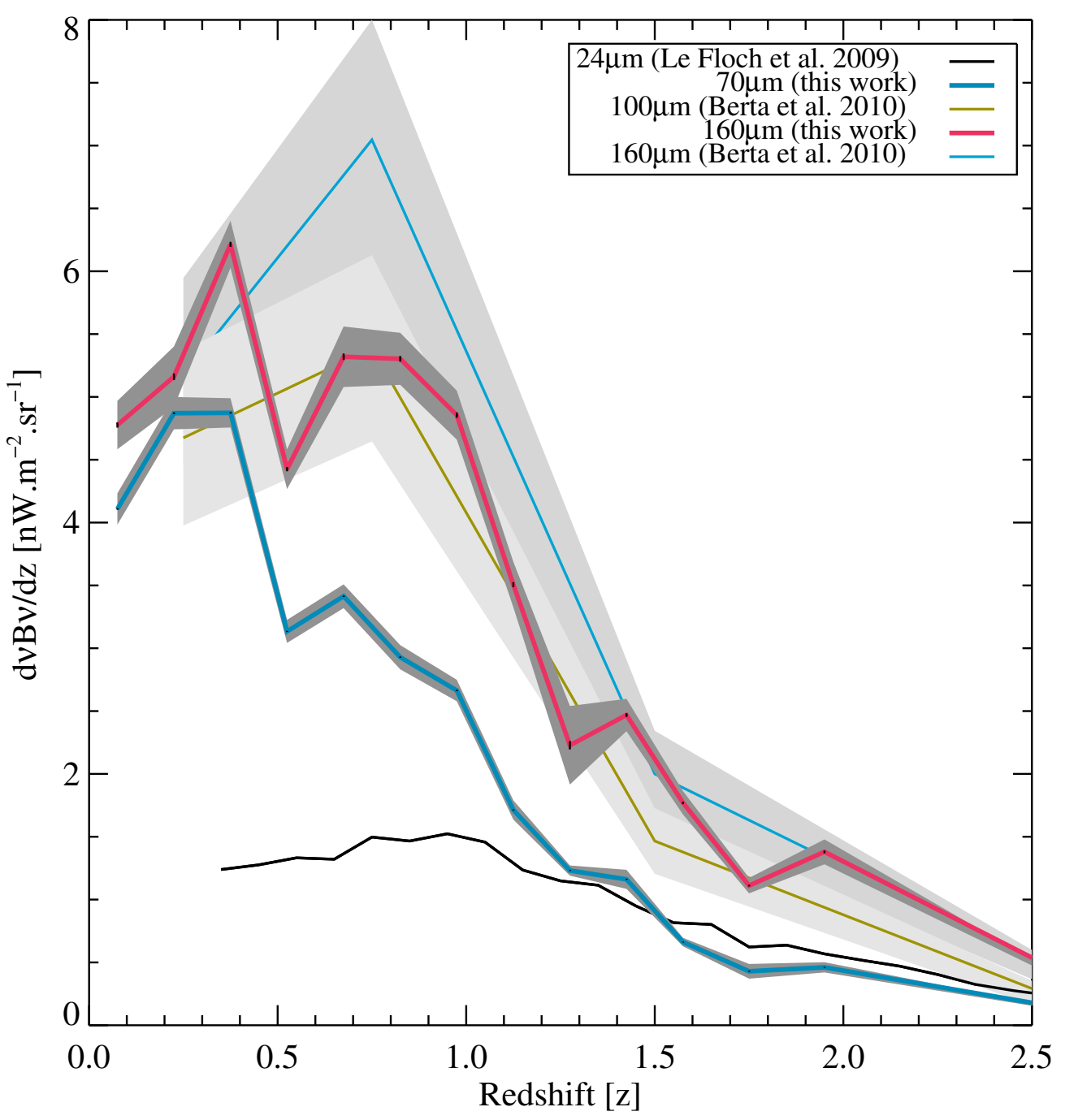

Fig. 9. The cosmic infrared background history since $z \sim 2$ at 24, 70, 100, and $160 \mu \mathrm{m}$ as measured from galaxies selected at $24 \mu \mathrm{m}$ with $S_{24}>80 \mu \mathrm{Jy}$. The $24 \mu \mathrm{m}$ data come from Le Floc'h et al. (2009), and the 70 and $160 \mu \mathrm{m}$ data from this work, all using the Spitzer/MIPS data of COSMOS field. The 100 and $160 \mu \mathrm{m}$ data in four redshift bins are from Berta et al. (2010) in GOODS-N with Herschel/PACS (using the same $24 \mu \mathrm{m}$ prior.).

is flatter (or broader) with redshift, and its greatest contribution is from sources at around $z \sim 1$. This mid-infrared distribution has a relative contribution at $z>1.5$ larger than in the far-IR, i.e., the decay slope is shallower at $24 \mu \mathrm{m}$ than at $70 \mu \mathrm{m}$ and longer wavelengths.

A detailed comparison, however, is still difficult because of cosmic variance. The results from Berta et al. (2010) are based on GOODS-N, which has an area about 50 times smaller than considered here. We have furthermore shown that large-scale structure is visible at $z<0.5$ in the COSMOS field.

That most of the CIB between 100 and $160 \mu \mathrm{m}$ is identified as being produced by $z<1$ sources agrees with expectations (e.g. Lagache et al. 2005; Bethermin et al. 2010c). The $24 \mu \mathrm{m}$ background contains, however, a significant contribution from galaxies at $z>1$ (30\%, according to Le Floc'h et al. 2009). As expected and observed (Marsden et al. 2009; Pascale et al. 2009), most of the submillimeter background consists of sources at higher redshifts $(z>1.5)$. Other analyses found conflicting results (see Wang et al. 2006; Serjeant et al. 2008). This wavelength versus redshift dependence of the background can be explained by analyzing the sum of the SED of galaxies at various redshifts, in particular the peak emission in the far-infrared of the reprocessed starlight by dust that is redshifted. Thus, the SED of the CIB is broader and flatter at far-infrared and submillimeter wavelengths than for any individual galaxy SED.

\subsection{The models}

These observations can be confronted to models. We considered three backward evolution models developed to fit infrared data: Lagache et al. (2004), Le Borgne et al. (2009), Valiante et al. (2009), and Bethermin et al. (2010c), among an abundant literature (for the most recent: Franceschini et al. 2008, 2009; Pearson \& Khan 2009; Rowan-Robinson 2009). The main feature of the Lagache et al. (2004) model is the use of two galaxy population spectral energy distributions (SEDs), normal/cold and starburst galaxies, parametrized by their total infrared luminosity. The local luminosity functions are fitted, and the evolution in redshift is applied to fit the additional constraints of the observed number counts, CIB SED, and CIB fluctuation level. 

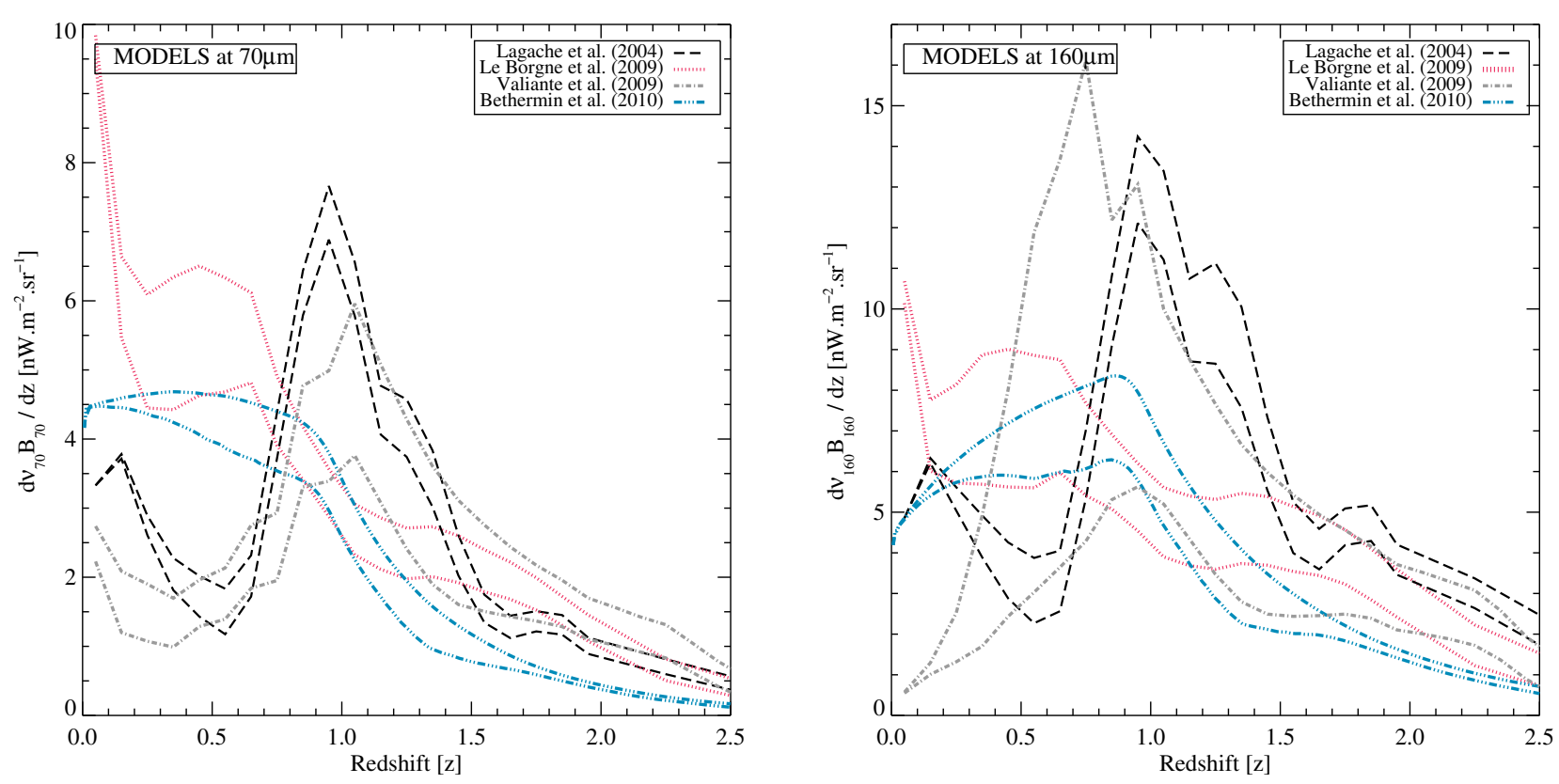

Fig. 10. Differential $70($ left $)$ and $160 \mu \mathrm{m}$ (right) background brightness $\mathrm{d} v_{v} B_{v} / \mathrm{d} z$ as a function of redshift, in units of $\mathrm{nW} \mathrm{m}^{-2} \mathrm{sr}^{-1}$, as predicted by 4 models: dash black line: Lagache et al. (2004) model; Dot magenta line: Le Borgne et al. (2009) model; Dot-dash grey line: Valiante et al. (2009); Dot-dot-dot-dash blue line: Bethermin et al. (2010c) model. For each model, the top line corresponds to all galaxies, and the bottom line to the prior selection (used in this work) of $S_{24}>80 \mu \mathrm{Jy}$.

The Le Borgne et al. (2009) model is based on an automated minimization of the difference between the model and selected datasets (local luminosity functions, number counts, CIB absolute level) with a given SED library (Chary et al. 2001). The Valiante et al. (2009) model introduces scatter into the SED by using Monte Carlo simulations for an extended library based on observations from the Spitzer archive, and contains starburst and AGN contributions as a function of IR luminosity. These three models used Spitzer data, and were developed prior to the availability of Herschel data. Finally, the Bethermin et al. (2010c) model is a fully-parametric approach, automatically fitting the latest Spitzer, BLAST \& Herschel data with the Markov chain Monte Carlo (MCMC) (Dunkley et al. 2005) method, and using the Lagache et al. (2004) SED library of two galaxy populations.

Figures 6 and 7 overplot these three models for a prior selection at $24 \mu \mathrm{m}$ (with $S_{24}>80 \mu \mathrm{Jy}$ ) to be consistent with the data we compare. Figure 10 shows the models, with this prior cut, but also without any cut, i.e. all the galaxies. The main differences can be summarized as follows:

- the Lagache et al. (2004) model predicts a contribution of infrared galaxies to the 70 and $160 \mu \mathrm{m}$ backgrounds peaking at $z \sim 1$, which is not observed; the predicted dip at $z \sim 0.5$ is also not observed;

- the Le Borgne et al. (2009) model overpredicts the galaxy contributions at $z \leq 0.3$, i.e., desagrees with observations, despite the poor statistical quality of the observations; the reason for this desagreement is probably the is likely a lack of a cold galaxy component at $z \sim 0$. The general shape of the model at $z>0.3$ agrees with the data, despite predicted peak and high-redshift $(z>1)$ tail being slightly higher than observed;

- the Valiante et al. (2009) model exhibits similar trends to that of Lagache et al. (2004): almost no contribution at low redshift (in disagreement with data at 70 and $160 \mu \mathrm{m}$ ), and a pronounced peak at $z \sim 1$, which is not observed. This models reproduces well, however, the $z>1$ tail at $160 \mu \mathrm{m}$ (but not at $70 \mu \mathrm{m}$ );

- the Bethermin et al. (2010c) model provides a closer fit to the data, most likely because its optimal use of Spitzer \& Herschel data (Bethermin et al. 2010a; Oliver et al. 2010) at far-infrared and submillimeter wavelengths already takes into account the statistical properties of galaxies in an empirical way;

- the selection of $S_{24}>80 \mu \mathrm{Jy}$ galaxies to estimate the background buildup with redshift produces an almost flat cut in redshift to the brightness (comparison of the two lines for each model in Fig. 10, except for Valiante et al. (2009) at $160 \mu \mathrm{m}$ with larger variations). Thus, the peak and the structure in redshift observed with the $S_{24}>80 \mu \mathrm{Jy}$ cut is not significantly affected by this selection, and our conclusions based on this cut can be extended to the whole CIB buildup, at least for $z<2.5$. However, the $S_{24}>80 \mu \mathrm{Jy}$ cut might cause a problem of strong incompleteness at Herschel SPIRE wavelengths, made-up by higher-redshift sources (Lagache et al. 2005; Marsden et al. 2009). A need to use fainter $24 \mu \mathrm{m}$ flux densities is thus required at submm wavelengths.

All these models predict similar star formation rates and evolutions of the luminosity function. Our work places stronger constraints on the models, which will have to fine-tune either the galaxies SED used or refine the luminosity function evolutions considered.

\section{Conclusion}

As shown by Le Floc'h et al. (2009) and our results, comparing the CIB buildup (differential brightness vs. redshift) with models allows us to constrain the parameters and break degeneracies, since models usually predict the same number counts and CIB SED, but different redshift histories for the luminosity functions for instance. Using exquisite Spitzer data on one of the widest and deepest fields, we have been able to infer that the maximum 
contributions to the $70 \mu \mathrm{m}$ background (when selecting $24 \mu \mathrm{m}$ galaxies with $S_{24}>80 \mu \mathrm{Jy}$ ) occur at both $z<0.5$ and $0.3<z<$ 0.9 for the $160 \mu \mathrm{m}$ background.

We have deduced that $74 \%$ of the $160 \mu \mathrm{m}$ background was emitted at $z \leq 1$, and $81 \%$ at $70 \mu \mathrm{m}$. We have also estimated the AGN contribution to the far-infrared background to be less than about $10 \%$ for $z<1.5$.

Results obtained for preliminary Herschel/PACS data on GOODS-N by Berta et al. (2010) agree with our findings, despite the uncertainties due to large-scale structure. This consistency with the results confirms that the stacking analysis method is a valid approach to estimate the components of the far-IR background using prior information about resolved mid-IR sources.

The Lagache et al. (2004) model predictions mainly disagree with data, since a peak in the contributions at $z \sim 1$ is not observed. The Le Borgne et al. (2009) model disagrees with the data at low redshift (most likely because of the SED used), but succeeds in reproducing most of the observed trend, despite there being an excess at $z>1.5$. The Bethermin et al. (2010c) model is consistent with the data.

Our study, combined with those of Le Floc'h et al. (2009) and Berta et al. (2010), can allow to place tighter constraints on the models of galaxy evolution, since their predictions can strongly vary with redshift, despite close fits of the number counts, luminosity functions, and cosmic infrared background spectral energy distributions.

This study, and forthcoming analyses of Herschel data, will also help to refine the models used to compute the far-infrared and submillimeter emissivity with redshift, which is needed to compute the optical depth for (hundreds of) TeV photons. Since the opacity of the Universe to $\mathrm{TeV}$ photons depends on the infrared luminosity density along the line of sight, the buildup history of the CIB has a direct effect on the TeV photon propagation. We have shown that most $(\sim 80 \%)$ of the far-infrared background is produced at $z<1$, in a regime where many blazars are observed (e.g. Aharonian et al. 2006; Albert \& Magic Collaboration 2008). The model predictions for TeV obscuration models (e.g. Mazin \& Raue 2007; Franceschini et al. 2008; Stecker \& Scully 2009; Kneiske \& Dole 2010; Younger \& Hopkins 2010; Bethermin et al. 2010c) can be distinguished at $z \leq 0.3$, where the CIB has the greatest contribution to high energy photons emission, by comparing their CIB buildup history with our data.

Acknowledgements. Part of this work was supported by the D-SIGALE ANR06-BLAN-0170 and the HUGE ANR-09-BLAN-0224-02. M. J. thanks the "Matière Interstellaire et Cosmologie" group at IAS and the CNRS for the funding. K. C. acknowledges founding from a Leverhulme Trust Early Career Fellowship. We thank S. Berta for providing us with an electronic form of his latest Herschel/PACS PEP published results, and D. Le Borgne and E. Valiante for the public access to their models. We thank D. Elbaz, E. Daddi, B. Magnelli, and R.-R. Chary for fruitful discussions. In memoriam, Pr. Ph. Jauzac (August 29th, 1948 - October 22nd, 2009) (M. J.).

\section{References}

Aharonian, F., Akhperjanian, A. G., Bazer-Bachi, A. R., et al. 2006, Nature, 440, 1018

Aharonian, F., Akhperjanian, A. G., Barres De Almeida, U., et al. 2007, A\&A, 475, L9

Albert, J., \& Aliu, E., \& Magic Collaboration 2008, Science, 320, 1752

Alonso-Herrero, A., Perez-Gonzalez, P. G., Alexander, D. M., et al. 2006, ApJ, 640,167
Bavouzet, N. 2008, Les galaxies infrarouges: distribution spectrale d'énergie et contributions au fond extragalactique, Ph.D. Thesis, Université Paris-Sud 11 http: //tel .archives-ouvertes . fr/tel-00363975/

Berta, S., Magnelli, B., Lutz, D., et al. 2010, A\&A, 518, L30

Bethermin, M., Dole, H., Beelen, A., et al. 2010a, A\&A, 512, A78

Bethermin, M., Dole, H., Cousin, M., \& Bavouzet, N. 2010b, A\&A, 516, A43

Bethermin, M., Dole, H., Lagache, G., Le Borgne, D., \& Penin, A. 2010c, A\&A, in prep.

Bower, R. G., Benson, A. J., Malbon, R., et al. 2006, MNRAS, 370, 645

Brusa, M., Zamorani, G., Comastri, A., et al. 2007, ApJS, 172, 353

Brusa, M., Civano, F., Comastri, A., et al. 2010, ApJ, 716, 348

Cappelluti, N., Hasinger, G., Brusa, M., et al. 2007, ApJS, 172, 341

Cappelluti, N., Brusa, M., Hasinger, G., et al. 2009, A\&A, 497, 635

Caputi, K. I., Dole, H., Lagache, G., et al. 2006, ApJ, 637, 727

Caputi, K. I., Lagache, G., Yan, L., et al. 2007, ApJ, 660, 97

Cattaneo, A., Faber, S., Binney, J., et al. 2009, Nature, 460, 213

Chary, R., \& Elbaz, D. 2001, ApJ, 556, 562

Chary, R., Casertano, S., Dickinson, M. E., et al. 2004, ApJS, 154, 80

Clements, D. L., Rigby, E., Maddox, S., et al. 2010, A\&A, 518, L8

Daddi, E., Alexander, D. M., Dickinson, M., et al. 2007, ApJ, 670, 173

Dole, H., Floc'h, E. L., Perez-Gonzalez, P. G., et al. 2004, ApJS, 154, 87

Dole, H., Lagache, G., Puget, J. L., et al. 2006, A\&A, 451, 417

Dunkley, J., Bucher, M., Ferreira, P. G., Moodley, K., \& Skordis, C. 2005, MNRAS, 356, 925

Dye, S., Dunne, L., Eales, S., et al. 2010, A\&A, 518, L10

Fernandez-Conde, N., Lagache, G., Puget, J. L., \& Dole, H. 2008, A\&A, 481, 885

Fernandez-Conde, N., Lagache, G., Puget, J. L., \& Dole, H. 2010, 515, A48

Ferrarese, L., \& Merritt, D. 2000, ApJ, 539, L9

Franceschini, A. G., Rodighiero, M., Vaccari, et al. 2008, A\&A, 487, 837

Franceschini, A., Rodighiero, G., Vaccari, M., et al. 2010, A\&A, 517, A74

Frayer, D. T., Fadda, D., Yan, L., et al. 2006a, AJ, 131, 250

Frayer, D. T., Huynh, M. T., Chary, R., et al. 2006b, ApJ, 647, L9

Frayer, D. T., Sanders, D. B., Surace, J. A., et al. 2009, AJ, 138, 1261

Hauser, M. G., \& Dwek, E. 2001, ARA\&A, 37, 249

Hauser, M. G., Arendt, R. G., Kelsall, T., et al. 1998, ApJ, 508, 25

Hogbom, J. A. 1974, ApJS, 15, 417

Hopkins, P. F., Hernquist, L., Cox, T. J., et al. 2006, ApJS, 163, 1

Hopkins, P. F., Younger, J. D., Hayward, C. C., Narayanan, D., \& Hernquist, L. 2010, MNRAS, 402, 1693

Ilbert, O., Capak, P., Salvato, M., et al. 2009, ApJ, 690, 1236

Kashlinsky, A. 2005, Phys. Rep., 409, 361

Kneiske, T. M., \& Dole, H. 2009, A\&A

Kneiske, T., \& Dole, H. 2010, A\&A, 515, A19

Lagache, G., Dole, H., Puget, J. L., et al. 2004, ApJS, 154, 112

Lagache, G., Puget, J. L., \& Dole, H. 2005, ARA\&A, 43, 727

Le Borgne, D., Elbaz, D., Ocvirk, P., \& Pichon, C. 2009, A\&A, 504, 727

Le Floc'h, E., Papovich, C., Dole, H., et al. 2005, ApJ, 632, 169

Le Floc'h, E., Willmer, C. N. A., Noeske, K., et al. 2007, ApJ, 660, L65

Le Floc'h, E., Aussel, H., Ilbert, O., et al. 2009, ApJ, 703, 222

Magnelli, B., Elbaz, D., Chary, R. R., et al. 2009, A\&A, 496, 57

Magorrian, J., Tremaine, S., Richstone, D., et al. 1998, AJ, 115, 2285

Marsden, G., Ade, P., Bock, J., et al. 2009, ApJ, 707, 1729

Mazin, D., \& Raue, M. 2007, A\&A, 471, 439

Oliver, S. J., Wang, L., Smith, A. J., et al. 2010, A\&A, 518, L21

Papovich, C., Dole, H., Egami, E., et al. 2004, ApJS, 154, 70

Pascale, E., Ade, P. A. R., Bock, J. J., et al. 2009, ApJ, 707, 1740

Pearson, C. S., \& Khan, A. 2009, MNRAS, 399, L11

Puget, J. L., Abergel, A., Bernard, J. P., et al. 1996, A\&A, 308, L5

Raue, M., Kneiske, T., \& Mazin, D. 2009, A\&A, 498, 25

Rieke, G. H., Young, E. T., Engelbracht, C. W., et al. 2004, ApJS, 154, 25

Rowan-Robinson, M. 2009, MNRAS, 394, 117

Salvato, M., Hasinger, G., Ilbert, O., et al. 2009, ApJ, 690, 1250

Serjeant, S. B. G., Dye, S., Peacock, J., et al. 2008, MNRAS, 386, 1907

Soifer, B. T., \& Neugebauer, G. 1991, AJ, 101, 354

Stecker, F. W., \& Scully, S. T. 2009, ApJ, 691, L91

Takeuchi, T. T., Ishii, T. T., Dole, H., et al. 2006, A\&A, 448, 525

Valiante, E., Lutz, D., Sturm, E., Genzel, R., \& Chapin, E. L. 2009, ApJ, 701, 74

Wang, W. H., Cowie, L. L., \& Barger, A. J. 2006, ApJ, 647, 1814

Werner, M. W., Roellig, T. L., Low, F. J., et al. 2004, ApJS, 154, 1

Younger, J. D., \& Hopkins, P. F. 2010, MNRAS, submitted [arXiv: 1003.4733 ] 